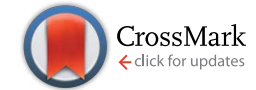

Cite this: RSC Adv., 2017, 7, 7901

Received 28th November 2016 Accepted 16th January 2017

DOI: 10.1039/c6ra27437d

www.rsc.org/advances

\section{Modification of thermal conductivity and thermal boundary resistance of amorphous Si thin films by Al doping}

\author{
Tianzhuo Zhan, ${ }^{\text {aa }}$ Masahiro Goto, ${ }^{a}$ Yibin $\mathrm{Xu},{ }^{a}$ Yohei Kinoshita, ${ }^{\mathrm{b}}$ Mamoru Ishikiriyama ${ }^{\mathrm{b}}$ \\ and Chikashi Nishimura ${ }^{a}$
}

\begin{abstract}
We investigate the effects of Al doping on the thermal conductivity and thermal boundary resistance of a-Si thin films. Au/Al-doped a-Si/Si structures were prepared by depositing Al-doped amorphous Si films of different Al doping concentrations and thickness on Si substrates by magnetron sputtering. The thermal resistances of the structures were measured to calculate the thermal conductivities of the films. The thermal conductivities of the $150 \mathrm{~nm}$-thick films were higher than those of $100 \mathrm{~nm}$-thick films, and a sharp increase in thermal conductivity with increasing Al doping concentration was observed in the $150 \mathrm{~nm}$-thick films but not in the $100 \mathrm{~nm}$-thick films. Furthermore, the thermal boundary resistances at the two interfaces in the structures also increased with increasing Al doping concentration. Our findings could be used to tailor the thermal resistance of materials for thermal management in semiconductor devices as well as for development of thermal barrier coatings and thermoelectric materials with good performance.
\end{abstract}

\section{Introduction}

Amorphous $\mathrm{Si}$ (a-Si) has previously been difficult to dope due to its high defect density, which has prevented the application of a-Si in semiconductor devices. ${ }^{1}$ Since the discovery of hydrogenated amorphous $\mathrm{Si}$ and the ability to efficiently dope this material, a-Si has become widely used in various semiconductor devices such as photovoltaic solar cells, thin-film transistors, image sensors, and data memories. ${ }^{2}$ For example, the use of a-Si thin films has enabled low-cost mass production of photovoltaic solar cells, because a-Si can be deposited at very low temperatures on inexpensive large-area substrates such as glasses and plastics. ${ }^{3}$ In addition to semiconductor device applications, a-Si also shows great potential in thermoelectric applications due to its low thermal conductivity. Although the electrical and optical properties of amorphous semiconductors have been studied extensively, the thermal properties have not been thoroughly investigated. ${ }^{4-7}$ Thermal transport in amorphous solids is quite different from crystalline materials, and the phonon-gas model is no longer valid in amorphous solids. Various theoretical models such as the Einstein model, ${ }^{8}$ the minimum thermal conductivity model, ${ }^{9}$ the fraction model, ${ }^{10}$ and the diffusion model ${ }^{11,12}$ have been proposed to describe thermal transport in amorphous solids. Despite these theoretical models, experimental studies on the thermal conductivities of amorphous solids are still limited. Furthermore, thermal

${ }^{a}$ National Institute for Materials Science, 1-2-1 Sengen, Tsukuba 305-0047, Japan. E-mail: ZHAN.Tianzhuo@nims.go.jp

${ }^{b}$ Toyota Motor Corporation, 1200, Mishuku, Susono-city, Shizuoka 410-1195, Japan boundary resistance, which plays an important role in nanostructured materials that have many interfaces ${ }^{13-15}$ is even less explored than thermal conductivity. Investigation of the thermal conductivity and thermal boundary resistance of a-Sibased materials is crucial for thermal management in semiconductor devices and for the development of efficient thermal barrier coatings and thermoelectric materials.

In this study, we investigate the effects of $\mathrm{Al}$ doping on the thermal conductivity and thermal boundary resistance of a-Si thin films. We deposited Al-doped a-Si thin films of different Al doping concentrations and thickness on $\mathrm{Si}$ substrate by magnetron sputtering and then deposited an Au film on top of the films. We then measured the thermal resistances of the $\mathrm{Au} /$ Al-doped a-Si/Si structures to calculate the thermal conductivities of the Al-doped a-Si thin films. We found that the thermal conductivities of $150 \mathrm{~nm}$-thick films are higher than those of the corresponding $100 \mathrm{~nm}$-thick films at the same Al doping concentration. Furthermore, as the Al doping concentration increases, a sharp increase in thermal conductivity is observed in the $150 \mathrm{~nm}$-thick films, but not in the $100 \mathrm{~nm}$-thick films. Furthermore, the thermal boundary resistances at the two interfaces in the structures also increase with increasing $\mathrm{Al}$ doping concentration. We discuss the underlying mechanisms of the results.

\section{Experimental}

Al-doped a-Si thin films were deposited on intrinsic single crystalline Si substrate $(0.5 \mathrm{~mm}$ thick) by RF magnetron sputtering using a laboratory-built combinatorial sputtering system 
with a floating potential during the coating process. ${ }^{\mathbf{1 6}}$ The vacuum chamber of the sputtering system was evacuated to under a base pressure of $5.0 \times 10^{-5} \mathrm{~Pa}$. The thin films were deposited at a low substrate temperature $\left(25{ }^{\circ} \mathrm{C}\right)$ in order to obtain amorphous films. The thin films were deposited using two magnetron sputter cathodes in the system. Sputtering targets of $\mathrm{Si}$ and $\mathrm{Al}$ (diameter: $50 \mathrm{~mm}$, thickness: $6 \mathrm{~mm}$, 99.999\% (Si) 99.9999\% (Al) purity, High Purity Chemical Co.) with argon gas (over 99.999\% purity) were used for sputtering. The low base pressure and high purity argon gas help prevent incorporation of impurities into the growing film. The working pressure of the gas was fixed at 0.4 Pa during the process, which was automatically controlled by a control gate valve combined with a capacitance manometer. The RF power for the sputter cathode of Si was fixed at $100 \mathrm{~W}$ and the power for $\mathrm{Al}$ was varied for changing the Al doping concentration. The sputter rate of $\mathrm{Si}$ and Al was precisely controlled by a crystal membrane microbalance monitor. The distance between the sample and the targets of $\mathrm{Si}$ and $\mathrm{Al}$ were fixed at $173 \mathrm{~mm}$ and $200 \mathrm{~mm}$, respectively. The $2-3 \mathrm{~nm}$ thick native oxide layers on the $\mathrm{Si}$ substrates were not removed prior to deposition of the Al-doped a-Si thin films. The Al doping concentrations of the films were $1.75 \%, 5 \%$, and $10 \%$. The $\mathrm{Al}$ doping concentration was varied to alter the microstructure of the amorphous thin films and the interfacial properties of the two interfaces. The film thicknesses were 50, 100, and $150 \mathrm{~nm}$ at each $\mathrm{Al}$ doping concentration. A 150 $\mathrm{nm}$ thick Au film was then deposited on the Al-doped a-Si films at $25{ }^{\circ} \mathrm{C}$ by magnetron sputtering to act as a laser absorber and temperature sensor.

The thermal resistances of the Au/Al-doped a-Si/Si structures were measured by using the frequency domain thermoreflectance method $(\omega$ method) under vacuum $(<0.02 \mathrm{~Pa})$. The sample is set horizontally on the sample stage. The thermoelectric cooler system keeps the sample stage at a constant temperature $\left(25^{\circ} \mathrm{C}\right)$. The Au film was heated by a pump laser with angular frequency $\omega$; the pump laser is a multimode diode laser with a wavelength of $405 \mathrm{~nm}$ and an output power of 360 $\mathrm{mW}$. The pump laser is modulated by the TTL signal from the function generator built in the digital signal processor lock-in amplifier (Stanford SR8300). The amplitude of the ac-temperature response is about $1 \mathrm{~K}$. The temperature response at the surface of the Au film was measured by a probe laser by the thermoreflectance technique. The probe laser is a single mode diode laser with a wavelength of $635 \mathrm{~nm}$ and an output power of $5 \mathrm{~mW}$. The probe laser beams are detected by the photodiode sensor. Fig. 1 shows a schematic of the fabricated structure and the measurement technique. The temperature at the surface of the $\mathrm{Au}$ film $T(0)$ can be obtained using the one-dimensional heat conduction model by the following equation: ${ }^{17-19}$

$$
\begin{aligned}
\frac{T(0)}{q d_{0}}= & \frac{\mathrm{e}^{-i \frac{\pi}{4}}}{\sqrt{2 \lambda_{2} C_{2}}} \omega^{-\frac{1}{2}}+R_{01}+R_{12}+\left(1-\frac{\lambda_{1} C_{1}}{\lambda_{2} C_{2}}\right) \frac{d_{1}}{\lambda_{1}} \\
& +\left(1-\frac{\lambda_{0} C_{0}}{\lambda_{2} C_{2}}\right) \frac{d_{0}}{\lambda_{0}} .
\end{aligned}
$$

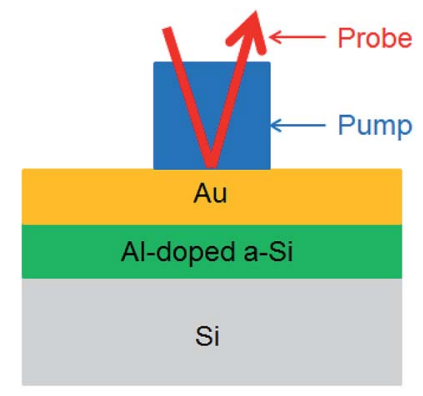

Fig. 1 Schematic illustration of the Au/Al-doped a-Si/Si structure and thermal conductivity measurement technique.

Here, $d$ is the film thickness, $q$ is the heat flux, $\lambda$ is the thermal conductivity, $C$ is the volumetric heat capacity. Subscripts 0,1 , and 2 denote the Au film, the Al-doped a-Si thin film, and the substrate, respectively, and $R_{01}$ and $R_{12}$ are the thermal boundary resistance at the Au/Al-doped a-Si thin film and Al-doped a-Si thin film/substrate interfaces, respectively. The fourth and fifth terms on the right-hand side are the thermal resistance of the Al-doped a-Si thin film and $\mathrm{Au}$ film, respectively. A plot of $T(0) /\left(q d_{0}\right)$ versus $\omega^{-1 / 2}$ gives a straight line where the $y$-intercept equals the total thermal resistance $R$ of the $\mathrm{Au} / \mathrm{Al}$-doped a-Si/Si structure. We measure $R$ of the samples with different Al-doped a-Si film thicknesses $d$, and plot $R$ as a function of $d$. The thermal conductivity of Al-doped a-Si thin film for various Al doping concentrations was calculated from the slope of the straight line fitted to the data points for each doping concentration. The slope of the line equals to $\frac{1}{\lambda_{1}}-\frac{C_{1}}{\lambda_{2} C_{2}}$, and with $C_{1}, C_{2}$, and $\lambda_{2}$ known, we can calculate the thermal conductivities of the films. The thermophysical properties of the materials used in this study are listed in Table 1. Although the thermal conductivity and volumetric heat capacity of Au film may have some changes depending on the microstructure of the film, it does not significantly affect the result of $R$ because the value of fifth term is on the order of $10^{-9} \mathrm{~m}^{2} \mathrm{~K} \mathrm{~W}^{-1}$, which is significantly smaller than $R$. The volumetric heat capacity of $\mathrm{Al}^{21}$ and $\mathrm{a}-\mathrm{Si}^{22}$ are 2.43 and $1.61 \times 10^{6} \mathrm{~J} \mathrm{~m}^{-3} \mathrm{~K}^{-1}$, respectively. The volumetric heat capacity of Al-doped a-Si films with Al doping concentration of $1.75 \%, 5 \%$ and $10 \%$ are calculated to $1.62,1.65$ and $1.69 \times 10^{6} \mathrm{~J} \mathrm{~m}^{-3} \mathrm{~K}^{-1}$, respectively. Measurements were carried out at angular frequencies of 1,2, 4 and $8 \mathrm{kHz}$ and at three different locations on each sample, with each measurement performed three times to reduce error.

\section{Results and discussion}

Fig. 2 shows the measured thermal resistances of the $\mathrm{Au} / \mathrm{Al}-$ doped a-Si/Si structures of different $\mathrm{Al}$ doping concentrations as a function of film thickness. For a fixed Al doping concentration, the three data points are expected to lie on a straight line if the films of different thicknesses have the same thermal conductivity. However, the data points for the $150 \mathrm{~nm}$-thick films deviate from the linear fits (dashed lines). This indicates that the 100 and $150 \mathrm{~nm}$-thick films have different thermal 
Table 1 Thermophysical properties of the materials used in this study

\begin{tabular}{llll}
\hline & Thermal conductivity $\left(\mathrm{W} \mathrm{m} \mathrm{K}^{-1}\right)$ & Volumetric heat capacity $\left(10^{6} \mathrm{~J} \mathrm{~m}^{-3} \mathrm{~K}^{-1}\right)$ & \multicolumn{1}{c}{ Thickness } \\
\hline Au film & 178 (ref. 20) & 2.47 (ref. 21) & $150 \mathrm{~nm}$ \\
Si substrate & 148 (ref. 21) & 1.66 (ref. 21) & $0.5 \mathrm{~mm}$ \\
Al-doped a-Si film & To be calculated & $1.62,1.65,1.69$ & $50,100,150 \mathrm{~nm}$
\end{tabular}

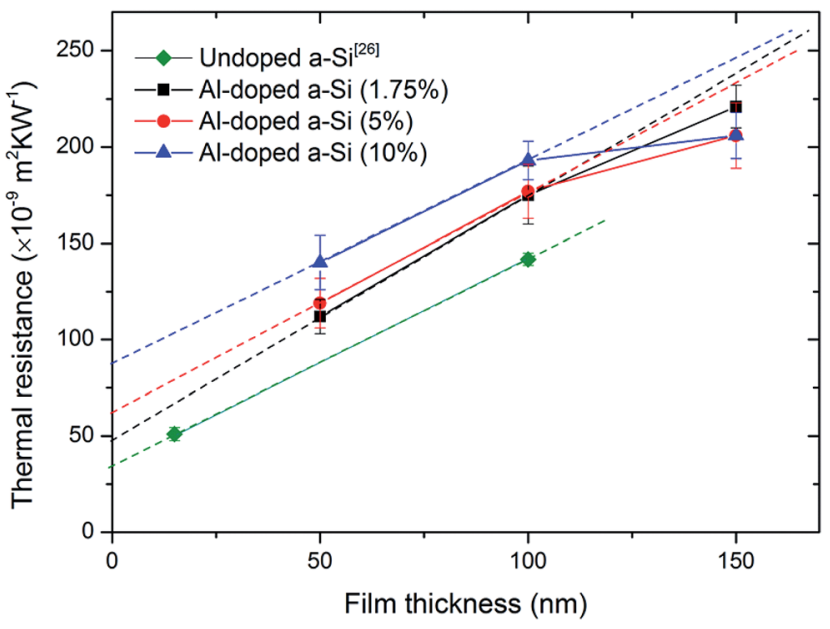

Fig. 2 Measured thermal resistance of Au/doped a-Si/Si structures with different $\mathrm{Al}$ doping concentration as a function of film thickness. Thermal resistances of the Au/a-Si/Si structures measured in our previous work are also included for comparison.

conductivities. This result is similar to the results for a-Si and aGe from previous reports. ${ }^{23-29}$ Previous work shows that the thermal conductivity of a-Si films with thicknesses less than 100 $\mathrm{nm}$ do not show observable film size dependence. ${ }^{24}$ If we assume this remains true for our Al-doped a-Si films, by fitting the two data points ( 50 and $100 \mathrm{~nm}, 50$ and $150 \mathrm{~nm}$ ) for each $\mathrm{Al}$ doping concentration, we can calculate the thermal conductivities of the 100 and $150 \mathrm{~nm}$-thick films. Fig. 3 shows the thermal conductivities of the 100 and $150 \mathrm{~nm}$-thick films as a function of Al doping concentration. It is evident that the thermal conductivities of the $150 \mathrm{~nm}$-thick films are higher than those of the corresponding $100 \mathrm{~nm}$-thick films at the same $\mathrm{Al}$ doping concentration. The $150 \mathrm{~nm}$-thick films also exhibit thermal conductivity that is more strongly dependent on Al doping concentration. The thermal conductivity in the $150 \mathrm{~nm}$-thick films increased sharply as the Al doping concentration increased, whereas the thermal conductivity in the $100 \mathrm{~nm}$ thick films increased only slightly. For comparison, the thermal conductivity of the $100 \mathrm{~nm}$-thick undoped a-Si thin film measured in our previous work ${ }^{26}$ was $0.93 \mathrm{~W} \mathrm{~m}^{-1} \mathrm{~K}^{-1}$, which is similar to the conductivity of the $100 \mathrm{~nm}$-thick Al-doped a-Si thin films.

We next discuss the underlying mechanisms of change in the thermal conductivity by doping with $\mathrm{Al}$. In semiconductors and insulators, heat is transported predominantly by phonons. However, doping with Al may modify the electrical conductivity of the a-Si thin films, and thus heat transport by electrons also needs to be considered. Although we did not measure the electrical conductivity of the Al-doped a-Si thin films, previous work $^{30}$ has reported that hydrogenated a-Si films with an $\mathrm{Al}$ doping concentration of $15 \%$ have an electrical conductivity of approximately $10^{-5} \Omega^{-1} \mathrm{~m}^{-1}$. This value is about two orders of magnitude higher than that of the undoped hydrogenated a-Si film, but still two orders of magnitude lower than that of crystalline Si (approximately $10^{-3} \Omega^{-1} \mathrm{~m}^{-1}$ ). The contribution of electrons to the heat transport in Al-doped a-Si can thus be ignored. For heat transport by phonons, thermal conductivity depends on how far phonons travel between scattering events, that is, on the mean free path (MFP) of the phonons. ${ }^{21}$ Thus, changes in the thermal conductivity of a-Si thin films may be induced by changes in the phonon MFP due to doping with Al. Previous works have shown that phonons with MFPs longer than $100 \mathrm{~nm}$ contribute to the thermal conductivity in a-Si. ${ }^{23-26}$ In 100 and $150 \mathrm{~nm}$-thick films, boundary scattering limits the MFPs of these phonons to $100 \mathrm{~nm}$ and $150 \mathrm{~nm}$, respectively. This could explain why the $150 \mathrm{~nm}$-thick films had a higher thermal conductivity than the $100 \mathrm{~nm}$-thick films. Furthermore, we observed that thermal conductivity in the $150 \mathrm{~nm}$-thick films increased sharply as the Al doping concentration increased. This indicates that doping with $\mathrm{Al}$ increased the number of phonons with MFPs longer than $100 \mathrm{~nm}$. However, in the 100 nm-thick films, the MFPs of these increased phonons are limited to $100 \mathrm{~nm}$ by boundary scattering. Furthermore, we observed that the measured thermal conductivity in the $100 \mathrm{~nm}$ thick films increased slightly as the Al doping concentration increased. This indicates that the effect of $\mathrm{Al}$ doping on the

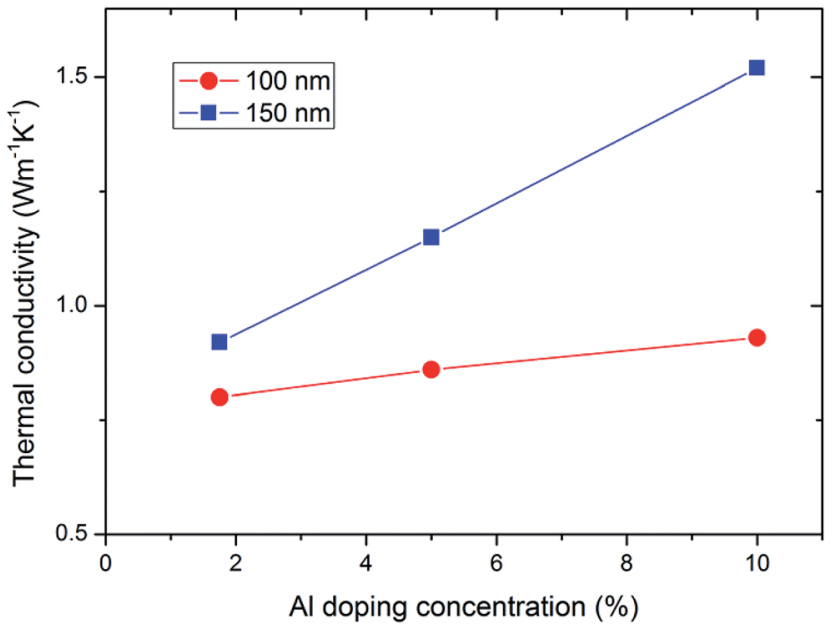

Fig. 3 Thermal conductivities of the 100 and $150 \mathrm{~nm}$-thick Al-doped a-Si films as a function of Al doping concentration. 
number of phonons with MFPs shorter than $100 \mathrm{~nm}$ is slight. This is in good agreement with the results of previous work, ${ }^{28}$ which found that only propagons (phonon-like propagating modes) with MFPs greater than $100 \mathrm{~nm}$ contribute significantly to thermal conductivity in a-Si. For films with thicknesses less than $100 \mathrm{~nm}$, the thermal conductivity is dominated by diffusons (non-propagating delocalized modes). Also, previous study showed that Ga atoms decreased the thermal conductivity of solid-phase epitaxy Si layer by acting as phonon scatters. ${ }^{31}$ By contrast; in our study the $\mathrm{Al}$ atoms played a totally different role in a-Si. $\mathrm{Al}$ atoms increased the thermal conductivity of a-Si thin films by increasing the number of phonons with long MFPs.

The thermal boundary resistances at the two interfaces of the structures are the intercepts of the linear fits (dashed lines in Fig. 2). It can be clearly seen that the thermal boundary resistances of Al-doped samples were higher than undoped samples and increase with increasing Al doping concentration. Thermal boundary resistance is attributed to the mismatch between the phonon spectra of the materials on both sides of the interface. The diffuse mismatch model (DMM) provides a quick and simple way to predict thermal boundary resistance from the mismatch between phonon spectra. The Debye temperature plays an important role in determining the phonon spectra in materials. Thus, materials on both sides of the interface with larger difference in Debye temperature typically have larger thermal boundary resistance. The Debye temperatures of a-Si and $\mathrm{Al}$ are $487 \mathrm{~K}$ (ref. 23) and $428 \mathrm{~K}$, respectively. The Debye temperature of Al-doped aSi films $\left(\mathrm{a}-\mathrm{Si}_{1-x} \mathrm{Al}_{x}\right)$ can thus be estimated by the equation $\theta_{\mathrm{D}}=$ $(487-59 x) \mathrm{K}^{32}$ When the $\mathrm{Al}$ doping concentration is $10 \%$, the Debye temperature of the Al-doped a-Si film is estimated to be $481 \mathrm{~K}$, indicating that the change in Debye temperature induced by doping with $\mathrm{Al}$ is extremely small. This indicates that the DMM model fails to explain the significant enhancement of the thermal boundary resistance. It is known that, in addition to the mismatch of phonon spectra, interfacial properties such as interfacial roughness, interdiffusion, bonding strength, and interface chemistry also have a considerable effect on thermal boundary resistance, ${ }^{33-36}$ and these effects are not considered in the DMM model. Thus, we speculate that the interfacial properties at the two interfaces were modified by doping with $\mathrm{Al}$, resulting in the enhancement of the thermal boundary resistance.

\section{Conclusions}

In summary, we investigated the effects of Al doping on the thermal conductivity and thermal boundary resistance of a-Si thin films. We found that the thermal conductivities of $150 \mathrm{~nm}$ thick films were higher than the corresponding $100 \mathrm{~nm}$-thick films with the same Al doping concentration, indicating that phonons with MFPs longer than $100 \mathrm{~nm}$ contribute to the thermal conductivity in Al-doped a-Si films. As the Al doping concentration increased, the thermal conductivities of the 150 nm-thick films increased sharply, a phenomenon that was not observed in the $100 \mathrm{~nm}$-thick films. This indicates that phonons with MFPs shorter than $100 \mathrm{~nm}$ are not greatly affected by $\mathrm{Al}$ doping. Furthermore, the thermal boundary resistance at the two interfaces also increased with increasing $\mathrm{Al}$ doping concentration. We speculate that the increase in the thermal boundary resistance is caused by modification of the interfacial properties at the two interfaces due to Al doping. The doping with Al modified both the thermal conductivity and thermal boundary resistance of the a-Si thin films, and this could be used to tailor the thermal resistance of a-Si based materials and structures for thermal management in semiconductor devices as well as for development of thermal barrier coatings and thermoelectric materials with good performance.

\section{References}

1 R. A. Street, Hydrogenated Amorphous Silicon, Cambridge University Press, 1991.

2 P. K. Shufflebotham, H. C. Card and A. Thanailakis, J. NonCryst. Solids, 1987, 92, 183-244.

3 A. Shah, J. Meier, A. Buechel, U. Kroll, J. Steinhauser, F. Meillaud, H. Schade and D. Domine, Thin Solid Films, 2006, 502, 292-299.

4 D. G. Cahill, M. Katiyar and J. R. Abelson, Phys. Rev. B: Condens. Matter Mater. Phys., 1994, 50, 6077-6081.

5 X. Liu, J. L. Feldman, D. G. Cahill, R. S. Crandall, N. Bernstein, D. M. Photiadis, M. J. Mehl and D. A. Papaconstantopoulos, Phys. Rev. Lett., 2009, 102, 035901.

6 B. L. Zink, R. Pietri and F. Hellman, Phys. Rev. Lett., 2006, 96, 055902.

7 R. Sultan, A. D. Avery, J. M. Underwood, S. J. Mason, D. Bassett and B. L. Zink, Phys. Rev. B: Condens. Matter Mater. Phys., 2013, 87, 214305.

8 A. Einstein, Ann. Phys., 1911, 35, 679-694.

9 G. A. Slack, in Solid State Physics: Advances in Research and Applications, ed. H. Ehrenreich, et al., Academic, New York, 1979, vol. 34, p. 1.

10 S. Alexander, O. E. Wohlman and R. Orbach, Phys. Rev. B: Condens. Matter Mater. Phys., 1986, 34, 2726-2734.

11 P. B. Allen and J. L. Feldman, Phys. Rev. Lett., 1989, 62, 645648.

12 J. L. Feldman, M. D. Kluge, P. B. Allen and F. Wooten, Phys. Rev. B: Condens. Matter Mater. Phys., 1993, 48, 12589-12602.

13 P. J. O'Brien, S. Shenogin, J. Liu, P. K. Chow, D. Laurencin, P. H. Mutin, M. Yamaguchi, P. Keblinski and G. Ramanath, Nat. Mater., 2013, 12, 118-122.

14 G. Pernot, M. Stoffel, I. Savic, F. Pezzoli, P. Chen, et al., Nat. Mater., 2010, 9, 491-495.

15 M. D. Losego, M. E. Grady, N. R. Sottos, D. G. Cahill and P. V. Braun, Nat. Mater., 2012, 11, 502-506.

16 M. Goto, A. Kasahara and M. Tosa, Appl. Surf. Sci., 2006, 252, 2482-2487.

17 Y. Xu, R. Kato and M. Goto, J. Appl. Phys., 2010, 108, 104317. 18 Y. Xu, M. Goto, R. Kato, Y. Tanaka and Y. Kagawa, J. Appl. Phys., 2012, 111, 084320.

19 T. Zhan, Y. Xu, M. Goto, Y. Tanaka, R. Kato and M. Sasaki, RSC Adv., 2015, 5, 49703-49707.

20 R. Kato and I. Hatta, Int. J. Thermophys., 2005, 26, 179-190. 
21 Japanese society of thermophysical, Thermophysical Properties Handbook, Japanese Thermophysical Society, Yokendo, Ltd, 1990.

22 B. L. Zink, R. Pietri and F. Hellman, Phys. Rev. Lett., 2006, 96, 055902.

23 X. Liu, J. L. Feldman, D. G. Cahill, R. S. Crandall, N. Bernstein, D. M. Photiadis, M. J. Mehl and D. A. Papaconstantopoulos, Phys. Rev. Lett., 2009, 102, 035901.

24 H. S. Yang, D. G. Cahill, X. Liu, J. L. Feldman, R. S. Crandall, B. A. Sperling and J. R. Abelson, Phys. Rev. B: Condens. Matter Mater. Phys., 2010, 81, 104203.

25 K. T. Regner, D. P. Sellan, Z. H. Su, C. H. Amon, A. J. H. McGaughey and J. A. Malen, Nat. Commun., 2013, 4, 1640.

26 T. Zhan, Y. Xu, M. Goto, Y. Tanaka, R. Kato, M. Sasaki and Y. Kagawa, Appl. Phys. Lett., 2014, 104, 071911.

27 T. Zhan, Y. Xu, M. Goto, Y. Tanaka, R. Kato, M. Sasaki and Y. Kagawa, AIP Adv., 2014, 4, 027126.

28 J. L. Braun, C. H. Baker, A. Giri, M. Elahi, K. Artyushkova, T. E. Beechem, P. M. Norris, Z. C. Leseman, J. T. Gaskins and P. E. Hopkins, Phys. Rev. B: Condens. Matter Mater. Phys., 2016, 93, 140201.

29 J. M. Larkin and A. J. H. McGaughey, Phys. Rev. B: Condens. Matter Mater. Phys., 2016, 89, 144303.

30 M. Yamaguchi, J. Non-Cryst. Solids, 1985, 77-78, 535-538.

31 T. Ueda, S. Sakane, T. Ishibe, K. Watanabe, S. Takeuchi, A. Sakai and Y. Nakamura, J. Electron. Mater., 2016, 45, 1914-1920.

32 F. Schaffler, in Properties of advanced semiconductor materials GaN, AlN, InN, BN, SiC, SiGe, ed. M. E. Levinshtein, S. L. Rumyantsev and M. S. Shur, John Wiley \& Sons, Inc., New York, 2001, pp. 149-188.

33 J. C. Duda and P. E. Hopkins, Appl. Phys. Lett., 2012, 100, 111602.

34 P. E. Hopkins, J. C. Duda, C. W. Petz and J. A. Floro, Phys. Rev. B: Condens. Matter Mater. Phys., 2011, 4, 035438.

35 J. C. Duda, C.-Y. P. Yang, B. M. Foley, R. Cheaito, D. L. Medlin, R. E. Jones and P. E. Hopkins, Appl. Phys. Lett., 2013, 102, 081902.

36 B. Gotsmann and M. A. Lantz, Nat. Mater., 2012, 12, 59-65. 